DOI: 10.2478/linpo-2013-0002

\title{
BEJA LEXICOSTATISTICS
}

\author{
VÁCLAV BLAŽEK
}

\begin{abstract}
Václav Blažek. Beja Lexicostatistics. Lingua Posnaniensis, vol. LV (1)/2013. The Poznań Society for the Advancement of the Arts and Sciences. PL ISSN 0079-4740, ISBN 978-83-7654-273-7, pp. 21-35.
\end{abstract}

The Beja (Bedawye) language is the only representative of the North Cushitic branch of the Cushitic languages. Although there are several dialects, e.g. Amar'ar, Arteiga, Beni Amer, Bishari, Hadendowa, Halenga etc., scholars collecting the lexical data of the Beja language usually do not distinguish between individual dialects and frequently summarize material of two or more dialects (e.g. Reinisch: Beni Amer, Bishari, Hadendowa), or they determine only the area, where their data were collected (e.g. Wedekinds: Eritrea; Hudson: Port Soudan and Tokar). Roper indicated the dialect Hadendowa, but according to Morin (1995: 22) it was a transitional interdialect of the Sinkat area. In this case it is impossible to separate specific lexicons of individual dialects and the only solution is to compare the lexical materials in dependence, who has collected them. Although there is only one distinctive phonetic isogloss dividing the Beja dialect continuum with typical $u$ in the north vs. $i$ in the south (VANHOvE 2006), the result of the present study demonstrates a relatively high internal diversity of the Beja lexicon. Two most incomplete or deviant sources, namely Munzinger and Bender, indicate the disintegration of common Beja to the 9th and 11th cent. respectively. The common share between the remaining idioms is $c .95 \%$ or higher, corresponding to the beginning of their disintegration around AD 1200 . This younger dating better agrees with at least partial intelligibility between the tribal dialects of Beja.

Václav Blažek, Masaryk University, Brno, Czech Republic, blazek@phil.muni.cz

The purpose of the present contribution is to confront the existing descriptions of the Beja lexicon. For practical reasons the comparisons are limited to the standard 100-wordlist. To ten richest lists, namely by Munzinger, Almkvist, Reinisch, Roper, Thelwall, Bender, Hudson, Starkey, Wedekind, Vanhove, the test of so-called 'recalibrated' glottochronology (see Starostin 1999/2000) was applied. Although their wordlists are usually not limited to the individual dialects, the authors usually described more dialects, the result can be interesting from the point of view of the internal diversity within the Beja dialect continuum. 
Table 1. Mutual percentages of common cognates on the basis of the standard 100-word-list, if loans are excluded

\begin{tabular}{|l|c|c|c|c|c|}
\hline \multicolumn{1}{|c|}{ Author $/ \%$} & Almkvist & Reinisch & Roper & Thelwall & Bender \\
\hline Munzinger & $75 / 79=94.9$ & $78 / 81=96.3$ & $74 / 85=87.1$ & $66 / 77=85.7$ & $65 / 76=85.5$ \\
\hline Almkvist & & $87 / 88=98.9$ & $83 / 88=94.3$ & $80 / 85=94.1$ & $72 / 80=90.0$ \\
\hline Reinisch & & & $93 / 97=95.9$ & $87 / 90=96.7$ & $77 / 83=92.8$ \\
\hline Roper & & & & $82 / 90=91.1$ & $76 / 84=90.5$ \\
\hline Thelwall & & & & & $68 / 79=86.1$ \\
\hline Bender & & & & & \\
\hline Hudson & & & & & \\
\hline Starkey & & & & & \\
\hline Wedekind & & & & & \\
\hline
\end{tabular}

\begin{tabular}{|l|c|c|r|c|}
\hline \multicolumn{1}{|c|}{ Author $/ \%$} & \multicolumn{1}{c|}{ Hudson } & \multicolumn{1}{c|}{ Starkey } & \multicolumn{1}{c|}{ Wedekind } & \multicolumn{1}{c|}{ Vanhove } \\
\hline Munzinger & $73 / 80=91.3$ & $68 / 73=93.2$ & $75 / 80=93.8$ & $78 / 82=95.1$ \\
\hline Almkvist & $81 / 85=95.3$ & $74 / 80=92.5$ & $82 / 86=95.3$ & $90 / 92=97.8$ \\
\hline Reinisch & $93 / 95=97.9$ & $80 / 84=95.2$ & $91 / 94=96.8$ & $94 / 96=97.9$ \\
\hline Roper & $91 / 95=95.8$ & $79 / 84=94.0$ & $89.5 / 93=96.2$ & $91 / 95=95.8$ \\
\hline Thelwall & $86 / 87=98.9$ & $73 / 80=91.3$ & $81.5 / 87=93.7$ & $87 / 90=96.7$ \\
\hline Bender & $73 / 82=89.0$ & $70 / 78=89.7$ & $72 / 84=85.7$ & $78 / 84=92.9$ \\
\hline Hudson & & $79 / 82=96.3$ & $88.5 / 92=96.2$ & $92 / 93=98.9$ \\
\hline Starkey & & & $84 / 87=96.6$ & $84 / 85=98.8$ \\
\hline Wedekind & & & & $91 / 93=97.8$ \\
\hline
\end{tabular}

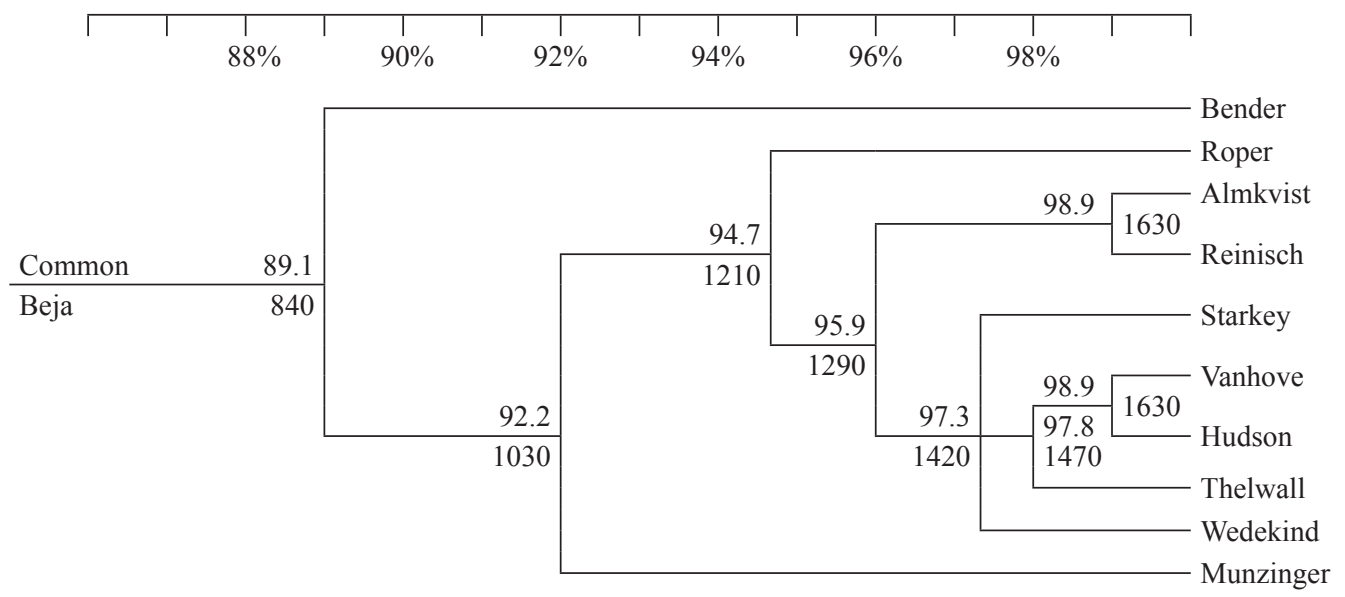

Tree diagram 1. The diagram depicts the internal diversity of the Beja dialect continuum constructed on the basis of Table 1 


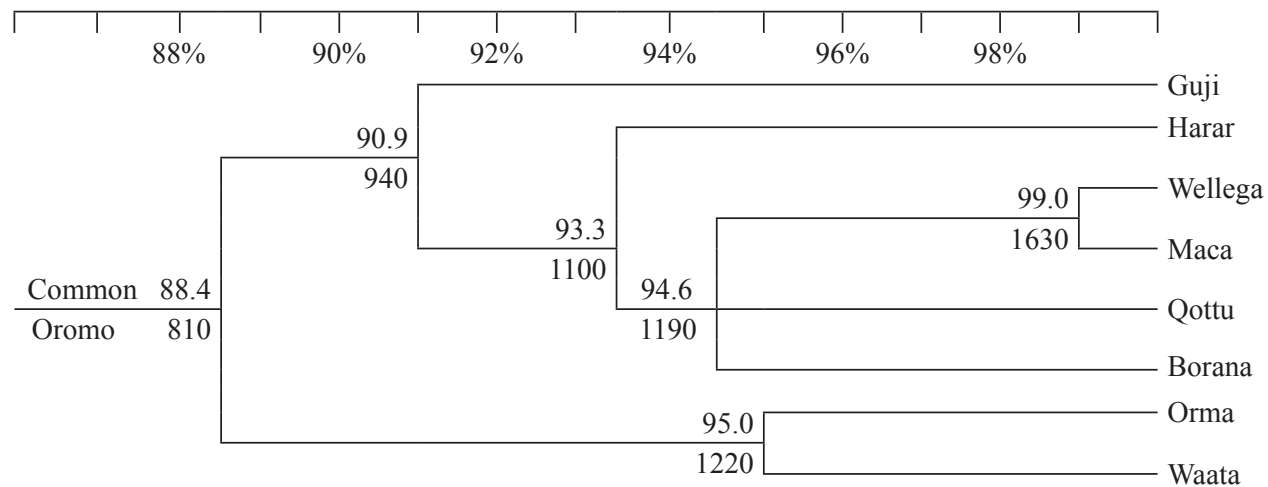

Tree diagram 2. The diagram depicts the result of the same test applied to Oromo dialects (BLAžEK 2010)

Appendix 1. Recent and (almost) complete wordlists

\begin{tabular}{|c|c|c|c|c|c|c|c|c|}
\hline $\begin{array}{l}\text { Author } \\
\text { gloss }\end{array}$ & $\begin{array}{c}\text { Reinisch } \\
1895\end{array}$ & $\begin{array}{c}\text { Roper } \\
1928\end{array}$ & $\begin{array}{c}\text { Thelwall } \\
1970\end{array}$ & $\begin{array}{c}\text { Bender } \\
1971\end{array}$ & $\begin{array}{c}\text { Hudson } \\
1995 / 64^{+}\end{array}$ & $\begin{array}{c}\text { Starkey } \\
\text { n.d. }\end{array}$ & $\begin{array}{l}\text { Wedekind } \\
2007\end{array}$ & $\begin{array}{c}\text { Vanhove } \\
2008\end{array}$ \\
\hline 1a. all & $\begin{array}{l}\text { káris } \\
\text { kars, kass }\end{array}$ & $\begin{array}{l}\text { kars } \bar{u} /-s s \bar{u} \\
\text { kissū }\end{array}$ & kisse & kasse & $\begin{array}{l}\text { kasso } \mathrm{m} . \\
\text { kasto } \mathrm{f} . \\
\text { karis } \mathrm{pl} .\end{array}$ & $\begin{array}{l}\text { kassay } \\
\text { kastay }\end{array}$ & kassaah & kass- adv. \\
\hline $1 \mathrm{~b}$. & & & & & koiye $\bar{e}$ & & & \\
\hline $1 \mathrm{c}$. & & & & & & nagway & & \\
\hline $1 d$. & & & & & & & & $\begin{array}{l}k w a \text { tous } \\
k w o o t i \text { toutes }\end{array}$ \\
\hline $1 \mathrm{e}$. & & & & & & & & akaanaku \\
\hline 2a. ashes & $n e^{\prime}$ ét hāšs & n'ćt hāšs & nn'etaš & nee'taš & nèt haš & naitaš & n'eetehaaš & n'eet-haaš \\
\hline $2 \mathrm{~b}$. & & & & & & torni & & \\
\hline 3a. bark & aḍif & aḍif & & aḍif & adif & adift & adift & 'adif \\
\hline $3 \mathrm{c}$. & éngili & & & & & & & \\
\hline $3 \mathrm{~d}$. & & & šaḍiiḍ & & šadid & & šaḍiiḍ & šadiid \\
\hline $3 e$. & & & & & & & & gaširi b., skin \\
\hline 4a. belly & $f i$ & $\begin{array}{l}\left(f(\tilde{l})^{\prime} i\right. \\
\text { stomach) }\end{array}$ & & & (fi' stomach) & $f e ̄ b$ & $o o-f^{\prime} i$ & (f'i stomach) \\
\hline $4 b$. & kálawa & $\begin{array}{l}\text { (kaláwa } \\
\text { interior) }\end{array}$ & kalawa & geléwa' & $\begin{array}{l}\text { (kilauwa } \\
\text { abdomen) }\end{array}$ & kalowb & u-kalawa & kaalawa \\
\hline $4 c$. & ésse & & & & & & & \\
\hline $4 d$. & & yam & & & $\begin{array}{l}\text { (iham, yam } \\
\text { womb) }\end{array}$ & yam & $\begin{array}{l}\text { (yaam } \\
\text { womb) }\end{array}$ & \\
\hline 5a. big & win/wun & win & wini & wIn & $\operatorname{win}^{+}$ & win & win & win \\
\hline $5 b .=49 c$ & & ragāāga & & & & ragaga & & \\
\hline 6a. bird & keláay & kélay & kilay & keláy & kilay & $k(e) l a i$ & kilaay & kiláy \\
\hline $6 \mathrm{~b}$. & sarî́n & & & & & & & \\
\hline 7a. bite & fenik & finik & fanniik & Effinık & fenik & ifni $k$ & finuukw & finik \\
\hline $7 \mathrm{~b}$. & tam $=23$ & & & & & & & \\
\hline 8a. black & hadál & hádal & hadali & hadél & hadal & hadal & hadal & hadal \\
\hline $8 \mathrm{~b}$. & & diri / dili & & & & & & $(\operatorname{diri}(f)$ \\
\hline $8 \mathrm{bc}$. & & dirif & & & & & & dark brown) \\
\hline 9. blood & bōy & boy & boi & boy & boi & boi & v. booyaa & booy \\
\hline 10. bone & mìta & mìtất & mmitat & mitat & mìtat & mitat & miitaatt & miitáat \\
\hline 11a. breast & & & & & & & & \\
\hline
\end{tabular}




\begin{tabular}{|c|c|c|c|c|c|c|c|c|}
\hline $\begin{array}{l}\text { Author } \\
\text { gloss }\end{array}$ & $\begin{array}{c}\text { Reinisch } \\
1895\end{array}$ & $\begin{array}{c}\text { Roper } \\
1928 \\
\end{array}$ & $\begin{array}{c}\text { Thelwall } \\
1970\end{array}$ & $\begin{array}{c}\text { Bender } \\
1971\end{array}$ & $\begin{array}{c}\text { Hudson } \\
1995 / 64^{+}\end{array}$ & $\begin{array}{c}\text { Starkey } \\
\text { n.d. }\end{array}$ & $\begin{array}{c}\text { Wedekind } \\
2007\end{array}$ & $\begin{array}{c}\text { Vanhove } \\
2008\end{array}$ \\
\hline 11a. & $n \bar{u} g$ & (nugw teat) & nnigw & & $n \breve{u} w k w$ & nig, negwab & nigw & nigw \\
\hline $11 \mathrm{~b} .=40$ & ginh/'a & & ginha & & & & & ginha \\
\hline $11 \mathrm{c}$. & & $d a b a$ & & & & & & \\
\hline 11d. & & degát & & & & & digat & \\
\hline $11 \mathrm{e}$. & & & & tuutćb & & & & \\
\hline $11 \mathrm{f} .=11 \mathrm{~b} ?$ & & & & & & agra & & \\
\hline 12a. burn & luw & liw & eetliin & allúw & leūw & $\operatorname{lau}(w), \operatorname{liw}$ & lawa & liw \\
\hline $12 \mathrm{~b}$ & balōl & & & & & & & \\
\hline $12 \mathrm{c}$. & & & & & & borbia & & \\
\hline $12 \mathrm{~d}$. & & & & & & $t i b^{\prime}(a)$ & & \\
\hline 13a. claw & $n e^{\prime} \bar{a} f$ & $n(\partial)^{\prime}$ 'af & $n n^{\prime} a f$ & $n$ 'af & (naf nail) & naf & n'aaf & n'af \\
\hline $13 \mathrm{~b}$ & éša & & & & & & & \\
\hline 14a. cloud & lēso & & & lasáa & & & & lasi/lasu \\
\hline $14 \mathrm{~b}$. & áfra & afrad & & & affratt & & & \\
\hline $14 \mathrm{c}$. & šāy & & & & šŏwŏw & & & šuwaw \\
\hline $14 \mathrm{~d}$ & alák & & & & & & & \\
\hline $14 \mathrm{e} .=\mathrm{f}+\mathrm{d}$ & ba'elík & & & & & & b'aluuk & b'alooki \\
\hline $14 \mathrm{f}$. & $b \bar{a} l$ & $b \bar{a} l$ & baal & & bal & & & bal/bali \\
\hline $14 \mathrm{~g}$. & mágwa & & & & & & & \\
\hline $14 \mathrm{~h}$. & gēm & & & & & & too-gim & \\
\hline $14 \mathrm{i}$. & $\underline{\operatorname{sah} a \dot{a} b}$ & $\underline{s a ̆ h a \bar{b} b}$ & & & & & & \\
\hline 15a. cold & lá'a & l'a & l'aabi & & $l a^{\prime}$ & láhab, ilāb & tu-l'aanaay & l'aa \\
\hline $15 \mathrm{~b}$. & & \begin{tabular}{|l|} 
'ákil \\
\end{tabular} & & & & & akil & \\
\hline $15 \mathrm{c}$. & & 'am'akwera & & moḳwaráab & & akwara & um'akwara & m'akwara \\
\hline 16a. come & $(y) i^{\prime}$ & $i$ & & $\varepsilon ’ a$ & $i$ & iy & $y i^{\prime} a a$ & ' $i$ \\
\hline $16 \mathrm{~b}$. & má'a! & & $m^{\prime} a$ & & $m a !$ & $m a^{\prime} a$ & m'aa & $m^{\prime} a$ \\
\hline $16 \mathrm{c}$. & & & & & $m-s-s^{+}$ & & & \\
\hline 17a. die & $y \bar{a}{ }^{\prime} i$ & $y a(y)$ & iyaai & iyá' & $\begin{array}{l}\text { yaiy }= \\
-y-y^{+}\end{array}$ & & & yaay \\
\hline $17 \mathrm{~b}$ & & & & & $n-s-' s$ & insis & misisa & misis \\
\hline 18a. dog & $y \bar{a} s$ & $y \bar{a} s$ & yas & syáas & yas & oyás & yaas & yaas \\
\hline $18 \mathrm{~b}$ & & & & & & oghūs & & \\
\hline 19a. drink & $g w^{\prime}$ & $g w^{\prime}(a)$ & gwiini & $g^{w}$ วyć' & $g w a$ & gwa! & $g w^{\prime} a a$ & $g w^{\prime} a$ \\
\hline 19aa. & & & & & & gwir & & \\
\hline $19 b$. & & gibit & & & & & gibita & $\begin{array}{l}\text { (gibit } \\
\text { drink milk) }\end{array}$ \\
\hline $19 \mathrm{c}$. & & & & & $\check{s}-f-y^{+}$ & šifōy & & $\begin{array}{l}\left(\begin{array}{l}\text { šifi } \\
\text { drink milk) }\end{array}\right. \\
\end{array}$ \\
\hline 20a. dry & & $\varepsilon \check{s} a$ & & & & $e \check{s} a, \overline{o s} i a$ & & eešaan \\
\hline $20 \mathrm{~b}$ & bálama & balama & balamaabi & balamáa & balam $\mathrm{v}$. & bal(amab) & balamaa & balama \\
\hline $20 \mathrm{c}$. & & & & & amar & & & \\
\hline $20 \mathrm{~d}$. & & & & & & letit & & \\
\hline $20 \mathrm{e}$. & & & & & & & & dimma \\
\hline 21. ear & angwìl & angwìl & aygwiil & angwiil & angwīl & angwil & angwiil & 'angwiil \\
\hline 22a. earth & $b \bar{u} r$ & $b \bar{u} r$ & & & bur & & buur & \\
\hline 22aa. earth & & & & & & & barar & \\
\hline $22 \mathrm{~b}$ & & $h \bar{a} \bar{s}=70$ & haaš & & haš & haš & (haaš soil) & haaš \\
\hline $22 \mathrm{c}$. & & & & & & daiyay & $\begin{array}{l}\text { (tu-dayaay } \\
\text { ground) }\end{array}$ & daayaay \\
\hline
\end{tabular}




\begin{tabular}{|c|c|c|c|c|c|c|c|c|}
\hline $\begin{array}{l}\text { Author } \\
\text { gloss }\end{array}$ & $\begin{array}{c}\text { Reinisch } \\
1895\end{array}$ & $\begin{array}{c}\text { Roper } \\
1928\end{array}$ & $\begin{array}{c}\text { Thelwall } \\
1970\end{array}$ & $\begin{array}{c}\text { Bender } \\
1971\end{array}$ & $\begin{array}{c}\text { Hudson } \\
1995 / 64^{+}\end{array}$ & $\begin{array}{l}\text { Starkey } \\
\text { n.d. }\end{array}$ & $\begin{array}{l}\text { Wedekind } \\
2007\end{array}$ & $\begin{array}{c}\text { Vanhove } \\
2008\end{array}$ \\
\hline $22 \mathrm{~d}$. & & & & & & (ard land) & & ('ard land) \\
\hline $22 \mathrm{e}$. & & & & & & & & tiin \\
\hline 23a. eat & tam & tam & tamiini & temmíia & tam & $\operatorname{tam}\left(a^{2}\right)$ & tamisama & tam \\
\hline $23 \mathrm{~b}$. & am & 'àm & & & & & & $\begin{array}{l}\text { ('aam } \\
\text { devour) }\end{array}$ \\
\hline 24. egg & $k w(a) h i$ & $k u h \bar{\imath} / k h w \bar{\imath}$ & kwiib & kwiip & $\begin{array}{l}\text { kwahi, } \\
\text { acc. } k w h a \bar{b} b\end{array}$ & $\begin{array}{l}k w \bar{e} b \\
\text { pl. qwīb }\end{array}$ & oo-kwhi & $k w h i$ \\
\hline 25a. eye & gwad/j & gw'ad & & & gwod & egwad & $g w^{\prime} a j$ & gw'ad pl. \\
\hline $25 \mathrm{~b}$ & lilí & lìlì & liiliib & leelit & lìli & liiliii/(o) & tu-liili & liili \\
\hline 26a. fat $n$. & semúm & & & & & & simuum- $t$ & sumum \\
\hline $26 \mathrm{~b}$. & $l \bar{a}$ & l'a & & & & & & l'aa \\
\hline $26 \mathrm{c}$. & dẹh & ḍha & dhaab & & dah v. & & (dha- $a b$ adj.) & (dha gros) \\
\hline $26 \mathrm{~d}$. & émfu & & & & & & & \\
\hline $26 \mathrm{e}$. & & & & goob & $g \bar{o} b$ & & & \\
\hline 27a. feather & & & & & $\begin{array}{l}\text { anbir / } \\
\text { ambir }\end{array}$ & $\begin{array}{l}\text { kilayi } \\
\text { amboor }\end{array}$ & & 'ambuur \\
\hline $27 \mathrm{~b}$. & $\underline{r \bar{s}}$ & & riǐs & & & $\underline{\text { rieš }}$ & riǐs & \\
\hline $27 \mathrm{c}$. & hámo $=36$ & & & & & & & \\
\hline $27 \mathrm{~d}$. & & timba & & & & & $\tan / \mathrm{mba}-a t$ & \\
\hline $27 \mathrm{e}$. & & $l \bar{a} t=46 \mathrm{a}$ & & & & & & \\
\hline 28. fire & n'a, n'e & n'e & nn'eet & ne'et & $n^{\prime} a$ & nait & too- $n$ 'i & $n^{\prime} i$ \\
\hline 29a. fish & $h \bar{u} t$ & & & & hut & & & \\
\hline $29 \mathrm{~b}$ & ášale & $\bar{a} \breve{s i}$ & $a a s ̌ o b$ & $a \check{s ̌ s ̌ a ́ b}$ & ašo & $a \check{s} o o b$ & w'aašu & 'aaši $i$ \\
\hline 30a. fly v. & fir & & & & & & firaa & \\
\hline $30 \mathrm{~b}$. & $b \bar{i} r$ & bir'īk & birr'ikiini & & $\begin{array}{l}\text { bir } \\
\text { birrik }\end{array}$ & obearti & \begin{tabular}{|l} 
biiraa \\
biir'iikaa
\end{tabular} & bar'iik \\
\hline $30 \mathrm{c}$. & kil & & & & & & & \\
\hline 30d. & serāa $r \rightarrow 6 \mathrm{~b}$ & & & & & & & \\
\hline 31a. foot & & & & & sukena & sikwina & & \\
\hline $31 \mathrm{~b}$ & ragád & ragad & & regéd & ragad & r/lagad & ragad & ragád \\
\hline $31 \mathrm{c}$. & & & dambet & & & $\begin{array}{l}\text { (dambe } \\
\text { sole })\end{array}$ & (danbi sole) & \\
\hline 32a. full & $a t \overline{a ́ b}$ & $a ́ t \bar{a} b$ & ataabi & & $a t \bar{a} b$ & atart? & ataab & 'atáab \\
\hline 32b. full & & & & & & & & amrama \\
\hline 32c. full & & & & & & & & 'atil v. \\
\hline 33a. give & $h i$ & $h i(w)$ & iniiyu & yihi' & $h i$ & & hiya & hiw \\
\hline $33 \mathrm{~b}$. & $n \bar{u} n$ & $n \bar{u} n$ & & & nun & nunia & nuипаa & nuиn \\
\hline 34a. good & šebốb & šibi & & & šibōb & & šibooba $\mathrm{v}$. & šiboob v. \\
\hline $34 \mathrm{~b}$. & (en) day & $d \bar{a}(y)$ & dyiibi & dayib & enday & dai $(-b / t)$ & & daa / daayi \\
\hline $34 d$. & (en)dáwre & & & & & & & \\
\hline $34 \mathrm{e}$. & adil & & & & & & & \\
\hline $34 \mathrm{f}$. & & & & & & gadut & & \\
\hline 35. green & sótay/-d- & sota & sootai & & $\begin{array}{l}\text { so:tă:y }{ }^{+} \\
\text {(sata } \\
\text { vegetation) }\end{array}$ & sot'aiy & sootaay & sootay \\
\hline 36a. hair & hámo & hami & hamot & hemóob & hamo & $\begin{array}{l}\text { hamaut } \\
\text { tihamu }\end{array}$ & tu-hamu & $\begin{array}{l}\text { hami / } \\
\text { hamoot }\end{array}$ \\
\hline $36 \mathrm{~b}$. & & & & & & hardugwi & & \\
\hline $36 c$. & & & & & & tifta & & \\
\hline 37a. hand & ay & cyi / ayi & ayeeb & ayćcb & aiy & (aeyi arm) & w-'ayi & 'ay \\
\hline
\end{tabular}




\begin{tabular}{|c|c|c|c|c|c|c|c|c|}
\hline $\begin{array}{l}\text { Author } \\
\text { gloss }\end{array}$ & $\begin{array}{c}\text { Reinisch } \\
1895\end{array}$ & $\begin{array}{c}\text { Roper } \\
1928 \\
\end{array}$ & $\begin{array}{c}\text { Thelwall } \\
1970\end{array}$ & $\begin{array}{c}\text { Bender } \\
1971\end{array}$ & $\begin{array}{l}\text { Hudson } \\
1995 / 64^{+}\end{array}$ & $\begin{array}{c}\text { Starkey } \\
\text { n.d. }\end{array}$ & $\begin{array}{c}\text { Wedekind } \\
2007\end{array}$ & $\begin{array}{c}\text { Vanhove } \\
2008\end{array}$ \\
\hline $37 \mathrm{~b}$. & kákwi & & & & & & & \\
\hline $37 \mathrm{c}$. & & & & & & kufa & & \\
\hline $37 \mathrm{~d}$. & & & & & & owšiba? & & \\
\hline 38a. head & gírma/gúo & girma & girmaab & girmá & gūrma & $\operatorname{agir}(i) m a$ & $u$-girma & girma \\
\hline $38 \mathrm{~b} .=55 \mathrm{a} ?$ & & & & & & rhiba & & \\
\hline 39. hear & māsuw & $m a \bar{a} s i(w)$ & eemsiu & maasu & masŭw & & maasiwa & maasiw \\
\hline 40a. heart & ginh/'a & gin'a & gin'a & gInáa' & gina & gin'a & gina' & gin'a \\
\hline 40b. & $\underline{\underline{e} b}$ & & & & & & & \\
\hline 40c. $=40 \mathrm{a} ?$ & & & & & & ogria & & \\
\hline 41a. horn & $d a^{\prime} / d a^{\prime}$ & $d^{\prime} a$ & d'aab & $d a \underline{a} b$ & $d a$ & & $o o-d^{\prime} a$ & $d^{\prime} a$ \\
\hline 41b. & $k \bar{o} s$ & & & & & & & \\
\hline 42. I & aní, áne & ane & aneeb & апии & ani & 'anēb & ani & áni \\
\hline 43. kill & dir & dir & darri & $I d I^{\prime} r$ & dir & $d^{\prime} i r$ & dira & dir \\
\hline 44a. knee & gúnba/-m- & gumba & & & $g \bar{u} m b a$ & gumba & oo-gwumba & $g(w) u m b a ́$ \\
\hline $44 \mathrm{~b}$ & gundíf & & gindif & & gundif & gindif & gindif & gandif \\
\hline 45a. know & & & ikteeni & & & iktaīn & & \\
\hline 45aa. & kan & kan & & Ikén & kan & ti-kane $2 \mathrm{sg}$ & kana & kan \\
\hline 46a. leaf & $r a \bar{t} t / l \bar{a} t$ & lāt & laat & laat & $\mathrm{rat} / \mathrm{lat}$ & lat, erat & raat & rat \\
\hline 46b. & báya & beyi/băyi & & & & & & \\
\hline 47a. lie & émbi' & $b^{\prime}(a)$ & n'atib'iini & & $b a$ & & b'iya & $b^{\prime} a$ \\
\hline $47 \mathrm{~b}$. & & & & & & & m'ana & \\
\hline 48a. liver & $s \bar{e}$ & se, si & seeti & & $s i$ & sait & too-si & $s i$ \\
\hline 48b. & & & & tonkola' & & & & \\
\hline 49a. long & $g^{w} \partial m \bar{a} d$ & gúmad & gumadi & & gumad & gwimud & gwimad & gwimád \\
\hline 49b. & serāra & sarāra & & & & sararab & saraaraab/t & saraar \\
\hline $49 \mathrm{c}$ & & & & ragáagab & & & & ragaag \\
\hline 50a. louse & se & & & & $\begin{array}{l}\text { (se camel } \\
\text { tick) }\end{array}$ & (sāt tick) & & \\
\hline $50 \mathrm{~b}$. & $t \bar{a} t$ & $t \bar{a} t$ & taat & taat & tat & & $\begin{array}{l}\text { (tateeyaa } \\
\text { be lousy) }\end{array}$ & tat pou \\
\hline $50 \mathrm{c}$ & & $\overline{i ́ b} a b$ & & & 'i:bă:b+ & & & \\
\hline 51a. man & tak & & tak & & $t a ́ k^{+}$ & tak & tak & tak \\
\hline $51 \mathrm{~b}$ & & rába & & & & & & (raba male) \\
\hline 52a. many & $g \dot{\bar{u}} d a$ & gwud & gwidaaba & gwodáab & gudāb & goodaba & $\begin{array}{l}\text { (too-gwud } \\
\text { plenty) }\end{array}$ & gwid v. \\
\hline 52b. many & & & & & & & & win $(n) e e t^{5 a}$ \\
\hline 53a. meat & $\check{s} a^{(())}$ & $\check{s} \bar{a}$ & šaat & & $\check{s} a$ & $\check{s} a$ & too-ša & $\check{s} a$ \\
\hline $53 \mathrm{~b}$ & & & & doof & & $d o f$ & & $\begin{array}{l}\text { (doof } \\
\text { morceau } \\
\text { de viande) }\end{array}$ \\
\hline 54a. moon & teríg & terig & eetrikt & terI'g & tirig & & (tirig month) & tirig \\
\hline $\begin{array}{l}55 \mathrm{a} . \\
\text { mountain }\end{array}$ & rééba/-i- & reba & $\begin{array}{l}\text { (ribaab } \\
\text { hill) }\end{array}$ & rebáb & rěba & rebab & $o o-r b a$ & riba \\
\hline $\begin{array}{l}55 \mathrm{~b} . \\
\text { mountain }\end{array}$ & konbúll & & & & & & kambuul & \\
\hline 56. mouth & $y \bar{a} f$ & yaf & yaf & $y \varepsilon f$ & $y \bar{e} f$ & yáf & yaf & yaf \\
\hline 57. name & $\mathrm{sim} / \mathrm{sum}$ & $\operatorname{sim}$ & $\operatorname{sim}$ & sIm & sim & oo-sim & $\operatorname{sim}$ & $\operatorname{sim}$ \\
\hline 58a. neck & ála & 'ála & alaat & $t^{\prime}$ ala' & alla & 'alat & tu-'ala & 'ala \\
\hline $58 \mathrm{~b}$ & máge & m'áge & & & m'aggi & m'ageb & & m'agi \\
\hline $58 \mathrm{c}$. & $m \bar{o} k$ & & & & & mokt & & \\
\hline
\end{tabular}




\begin{tabular}{|c|c|c|c|c|c|c|c|c|}
\hline $\begin{array}{c}\text { Author } \\
\text { gloss }\end{array}$ & $\begin{array}{c}\text { Reinisch } \\
1895\end{array}$ & $\begin{array}{c}\text { Roper } \\
1928\end{array}$ & $\begin{array}{c}\text { Thelwall } \\
1970\end{array}$ & $\begin{array}{c}\text { Bender } \\
1971\end{array}$ & $\begin{array}{c}\text { Hudson } \\
1995 / 64^{+}\end{array}$ & $\begin{array}{c}\text { Starkey } \\
\text { n.d. }\end{array}$ & $\begin{array}{l}\text { Wedekind } \\
2007\end{array}$ & $\begin{array}{c}\text { Vanhove } \\
2008\end{array}$ \\
\hline $58 \mathrm{~d}$. & $\mid \hat{e}$ & & & & & & & \\
\hline 59. new & $g \bar{a} y i$ & gey $\bar{\imath}$ & gayiibi & geyiib & $\begin{array}{l}\text { gayi }^{+} \\
\text {(gai' } i \text { now) }\end{array}$ & geyiny & gayiib/t & gayi \\
\hline 60a. night & hawád & hawād & hawaad & háwad & hauwād & hawadt & hawaad & hawáad \\
\hline 60b. & amás & & & & amass & & & $\begin{array}{l}\text { (amas } \\
\text { at night, } \\
\text { yesterday } \\
\text { night) }\end{array}$ \\
\hline $60 \mathrm{c}$. & tita & & & & & & & \\
\hline $60 \mathrm{~d}$. & & & & & akwit & & tu-'akwhi & $\begin{array}{l}\text { (akwhi } \\
\text { evening) }\end{array}$ \\
\hline 61. nose & genúf & ginù & ginuuf & $g \sigma n \sigma^{\prime} f$ & gěnūf & $o g(i) n u f$ & ginuuf & gunuuf \\
\hline 62a. not & $b a-$ & $b \bar{a} !$ & & & $b a-$ & $b \bar{a}$ & $b a-!$ & baa- \\
\hline $62 \mathrm{~b}$ & $k-$ & $k-$ & & & $k a$ & & $k-$ & $k a-/ k i-$ \\
\hline 63. one & $\begin{array}{l}\text { gāl/gārr } \\
\text { éngal/r }\end{array}$ & gal & gaali & ygá & gal/gar & $\begin{array}{l}\text { úngāal } \\
n g a a ̆ \text { : }\end{array}$ & $\begin{array}{l}\text { gaal } \\
\text { gaat }\end{array}$ & $\begin{array}{l}\text { gaal } \\
\text { gaat }\end{array}$ \\
\hline 64a. person & & & m'a adamiib & & adāni & & $\begin{array}{l}\text { m'aadamiib } \\
\text { women }+ \text { men }\end{array}$ & $\begin{array}{l}m \text { 'adáami }= \\
\text { women }+ \text { men }\end{array}$ \\
\hline $64 \mathrm{~b}$. & $h a$ & & & & & & & \\
\hline $64 c$. & pl. $d a$ men & pl. $d a$ men & & & $\begin{array}{l}\text { pl. děhay } \\
\text { people }\end{array}$ & $\begin{array}{l}\text { pl. dehá'y } \\
\text { people }\end{array}$ & $\begin{array}{l}\text { pl. dehay } \\
\text { people }\end{array}$ & \\
\hline 64d. & & tak $=51$ & & $t \varepsilon k$ & & & & \\
\hline 65a. rain n. & bire/-l- & bire & bireeb & bIréb & birri/billi & bireb & oo-bri & bíri r., sky \\
\hline $65 \mathrm{~b}$ & & & & & & mitwai & & \\
\hline 66. red & ádaro/-l- & adari/-li & adarob & adarób & adarōb & adaráb & adaroob/t & 'adaroo-b/t \\
\hline 67a. road & derááb $\underline{b}^{\circ} i b$ & darab & & & darab & darib & darab & darab \\
\hline $67 \mathrm{~b}$. & lági & lagi & & & leggi & & & \\
\hline $67 \mathrm{c}$. & sálla/sấâla & salal & salal & & sallal & & salal & \\
\hline $67 \mathrm{~d}$. & & & & sIkáb & & & & sikka \\
\hline $67 \mathrm{e}$. & & & & & $d o i$ & doy & & \\
\hline 68a. root & gadám & gádam & gadam & & gadum & radam & gadam & gadam \\
\hline $68 \mathrm{~b}$. & ádala & & & & & & & \\
\hline $68 \mathrm{c}$. & arấg & & & & & & & \\
\hline $68 \mathrm{~d}$. & & day & & & & & & daay \\
\hline $68 \mathrm{e}$ & & & & $\underline{s a r}$ & & & & \\
\hline 69a. round & debá̀la & & & & & & & \\
\hline $69 \mathrm{~b}$. & kwalál & kwádạd & & & $k^{w}-d-d^{+} \mathrm{v}$ & & kwalaal & $\begin{array}{l}\text { kwadaada } \\
\text { kwalaal }\end{array}$ \\
\hline 70b. sand & (h)issa (y) & asse / isse & asseet & & issi & & tu-'issi & 'assi \\
\hline $70 \mathrm{c} .=22 \mathrm{~b}$ & & & & haš & & & & haaš \\
\hline $70 \mathrm{~d}$ & & & & & & & & giisan \\
\hline 71a. say & di/yad & $d i$ & indi & 'ədor & $d i$ & di'abo & diya & $d i$ \\
\hline $71 \mathrm{~b}$. & & & & & & & & soodi \\
\hline $71 \mathrm{c}$. & an & & & & $n-y^{+}$ & & & $n$ \\
\hline 71d. & & & & & & $i h i$ & & \\
\hline 72a. see & erh & erh & rhiini & riyhah & rěh & & rhaa & rha \\
\hline $72 \mathrm{~b}$. & šebib & šibib & & & šibib & & & (šibib look) \\
\hline $72 \mathrm{c}$. & '⿳亠丷ara & & & & & & & \\
\hline $72 \mathrm{~d}$ & & & & & & hadwd? & & \\
\hline 73a. seed & derá⿱亠丷厂s / $\underline{s}_{-}$ & tera & teerab & terá & tora & & u-teera & teera \\
\hline
\end{tabular}




\begin{tabular}{|c|c|c|c|c|c|c|c|c|}
\hline $\begin{array}{l}\text { Author } \\
\text { gloss }\end{array}$ & $\begin{array}{c}\text { Reinisch } \\
1895\end{array}$ & $\begin{array}{c}\text { Roper } \\
1928 \\
\end{array}$ & $\begin{array}{c}\text { Thelwall } \\
1970\end{array}$ & $\begin{array}{c}\text { Bender } \\
1971 \\
\end{array}$ & $\begin{array}{c}\text { Hudson } \\
1995 / 64^{+}\end{array}$ & $\begin{array}{c}\text { Starkey } \\
\text { n.d. }\end{array}$ & $\begin{array}{c}\text { Wedekind } \\
2007\end{array}$ & $\begin{array}{c}\text { Vanhove } \\
2008\end{array}$ \\
\hline $73 \mathrm{~b}$. & & habba/hi- & & & $h a b b a$ & & tu-habba & \\
\hline $73 \mathrm{c}$. & & & & & bilay & & & \\
\hline 74a. sit & $s \bar{a}{ }^{\prime}$ & $s^{\prime}(a)$ & eest' $i$ & 'assá & $s \bar{a}=s-^{-}+$ & $s a^{\prime} a$ & $s a^{\prime} a$ & $s^{\prime} a$ \\
\hline $74 \mathrm{~b}$. & & & & & (fay be) & & & \\
\hline $74 \mathrm{c}$. & & & & & (hai be) & & & \\
\hline $74 \mathrm{~d}$. & kemis & & & & & & kimisa & \\
\hline $74 \mathrm{e}$. & & & & & & tațama & tațaa & \\
\hline 75a. skin & $a ́ d a$ & 'ade & & adeeb & addi & 'adēb & $w$-'adi & 'aade \\
\hline $75 \mathrm{~b}$. & & sar & & & $\operatorname{sár}^{+}$ & sir, sár & sar anim. & sar \\
\hline $75 \mathrm{c}$. & & gale & & & galō & & tu-galu & gali / gari \\
\hline $75 \mathrm{~d}$. & & & bašar & & & & & \\
\hline $75 \mathrm{e}$. & & & & & & & u-gana hum. & \\
\hline 76a. sleep & $d u w$ & diw & diwiini & dewiyáa & $d u(w), t u$ & & diwaa & daadiw \\
\hline $76 \mathrm{~b}$. & embi' $=47$ & & & & & & & $b^{\prime} a$ \\
\hline $76 \mathrm{c}$ & & & & & & naiy & naay & naa \\
\hline 77a. small & dis & disi & & & dis & diš & dis & dis \\
\hline $77 \mathrm{~b}$ & dábal/r/no & & dabaloob & deblúu & debalōb & dabalot & dabaloob/t & dabal,-i/-oo \\
\hline $77 \mathrm{c}$. & $d i$ & & & & & & & \\
\hline $77 \mathrm{~d}$. & & & & & enkalūs & & & \\
\hline $77 \mathrm{e}$. & & & & & enkąūut & & & \\
\hline $77 \mathrm{f}$. & & & & & & dimnut & & \\
\hline $77 \mathrm{~g}$. & & & & & & šilikt & & \\
\hline $77 \mathrm{~h}$. & & & & & & dagiga & & \\
\hline $\begin{array}{l}\text { 78a. smoke } \\
\text { n. }\end{array}$ & éga/-rd/d- & $\varepsilon ́ g a / \varepsilon d a$ & eeda & & $\bar{e} g a / \bar{e} d a$ & & $w$-'eega & 'eeda/'eega \\
\hline $78 \mathrm{~b}$. & & & & kwaraar & & & tu-kwaraar & \\
\hline $78 \mathrm{c}$. & & & & & & dorhan & & \\
\hline 79. stand & en-/negād & gad & ingadi & 'Ing'́cd & negad & inged & gada & (n) gad \\
\hline 80. star & hayúk & hayúk & hayuuk & hayúk & haiyūk & & hayiikw & hayuukw \\
\hline 81. stone & áwe & 'áwe & aweeb & aweeb & auwi & $\begin{array}{l}\text { 'oweb } \\
\text { ohawé }\end{array}$ & w'awi & 'awi \\
\hline 82. sun & $(y) \bar{i} n$ & $y \bar{y} n$ & yint & yiint & $y \overline{i n}$ & & too-yin & yiin \\
\hline 83a. swim & beḍf & & & & & & $\begin{array}{l}\text { (badif } \\
\text { swimming) }\end{array}$ & \\
\hline $83 \mathrm{~b}$. & $\overline{o m}$ & & uumiini & umiiya & & & uumaa & $\underline{\text { uum }}$ \\
\hline $83 \mathrm{c}$. & & & & & nidab & & & \\
\hline $83 \mathrm{~d}$. & & & & & & & kwibaha & $k w a b h$ \\
\hline $83 \mathrm{e}$ & & & & & & & bifa & \\
\hline 84a. tail & níwa & $n \bar{w} w a$ & niiwaab & niwa & $n \bar{\imath} w a$ & $\begin{array}{l}\text { niwab } \\
\text { onewa }\end{array}$ & tu-niiwa & niiwa \\
\hline 85. that & $b a-$ & $b \varepsilon-/ b a-$ & beeb & & $b \bar{e}-n-t$ & $b e-n /-t$ & $b e e-n /-t$ & $b e e-n /-t$ \\
\hline $\begin{aligned} \text { 86. this } & \text { sg. } \\
\text { m.: f. } & \text { pl. }\end{aligned}$ & $\begin{array}{l}\bar{u} n: t \bar{u} n \\
\bar{a} n: t \bar{a} n\end{array}$ & $\begin{array}{l}\bar{u} n: t \bar{u} n \\
\bar{a} n: t \bar{a} n\end{array}$ & oon & ons & & $\begin{array}{l}\bar{u} n: t \bar{u} n \\
\bar{a} n: \tan \end{array}$ & uun : tuun & $\begin{array}{l}\text { uun }: \text { tuun } \\
\text { aan }: \text { teen }\end{array}$ \\
\hline $\begin{array}{r}\text { 87. thou } \mathrm{m} . \\
\mathrm{f} .\end{array}$ & $\begin{array}{l}\text { barúk } \\
\text { batúk }\end{array}$ & $\begin{array}{l}\text { barūk } \\
\text { batūk }\end{array}$ & baruuk & berook & barūk & $\begin{array}{l}\text { barōk } \\
\text { batōk }\end{array}$ & baruuk & $\begin{array}{l}\text { barúuk } \\
\text { batúuk }\end{array}$ \\
\hline 88. tongue & mída(la) & mīdalāb & miidaab & midaláab & $\begin{array}{l}\text { mìdāb } \\
\text { midāla }\end{array}$ & $\operatorname{mid} a(l a) b$ & $\operatorname{miid}(a l) a a b$ & miidala \\
\hline 89a. tooth & $\begin{array}{l}k^{w} \text { ale } \\
k^{w} \text { are }\end{array}$ & kwire & kwiireeb & $k w I^{\prime} r c \varepsilon t$ & $k w i r i$ & quiraut & oo-kwri & $\begin{array}{l}\text { kwili dim. } \\
\text { kwiri }\end{array}$ \\
\hline $89 \mathrm{~b}$. & $k \bar{s} s$ & & & & & & & \\
\hline
\end{tabular}




\begin{tabular}{|c|c|c|c|c|c|c|c|c|}
\hline $\begin{array}{l}\text { Author } \\
\text { gloss }\end{array}$ & $\begin{array}{c}\text { Reinisch } \\
1895\end{array}$ & $\begin{array}{c}\text { Roper } \\
1928 \\
\end{array}$ & $\begin{array}{c}\text { Thelwall } \\
1970\end{array}$ & $\begin{array}{c}\text { Bender } \\
1971\end{array}$ & $\begin{array}{l}\text { Hudson } \\
1995 / 64^{+}\end{array}$ & $\begin{array}{l}\text { Starkey } \\
\text { n.d. }\end{array}$ & $\begin{array}{l}\text { Wedekind } \\
2007\end{array}$ & $\begin{array}{c}\text { Vanhove } \\
2008\end{array}$ \\
\hline $89 \mathrm{c}$. & nad & & & & & & & \\
\hline 89d. & & & & & $(d a$ molar $)$ & & & $\begin{array}{l}\left(d^{\prime} a a \text { molar }\right. \\
(n) d^{\prime} a \text { teeth) }\end{array}$ \\
\hline 90a. tree & hínde/i & hindi & hindib & Indiib & hindi & hindi & $w$-hindi & hindi \\
\hline 91. two & mál(l)o & mále & malooba & malósb & $\begin{array}{l}\text { mahló } \\
\text { mālō }\end{array}$ & mahlŏ: & maloob/t & $\begin{array}{l}\text { mhale } \\
\text { malli }\end{array}$ \\
\hline 92a. walk & $b \bar{a} y$ & $b a(y)$ & & & $b a i=b-y^{+}$ & & & bay \\
\hline $92 b$. & $g \bar{\imath} g$ & $g \bar{\imath} g$ & & giigy'i & $\begin{array}{l}\text { (gīg } \\
\text { go away) }\end{array}$ & giga & & $\begin{array}{l}\text { (giig } \\
\text { go away) }\end{array}$ \\
\hline $92 \mathrm{c}$. & sak & sak & & & $s a ́ k^{+}$ & saka & sakaa & sak \\
\hline $92 \mathrm{~d}$. & hirēr & hirer & hireeriini & & herēr & hirer & hiireeraa & hireer \\
\hline $92 \mathrm{e}$. & & dif & difa & & $\begin{array}{l}d \text {-' }^{+} \\
\text {(dif } \\
\text { go away) }\end{array}$ & difi & $\begin{array}{l}\text { (difa } \\
\text { go away) }\end{array}$ & $\begin{array}{l}\text { (dif } \\
\text { go away) }\end{array}$ \\
\hline $92 \mathrm{f}$. & & & & & & yeki & & \\
\hline $92 \mathrm{~g}$. & & & & & & dirink? & & \\
\hline $92 \mathrm{~h}$. & & & & & & ande I go & & \\
\hline 93a. warm & néb'a & $n a b^{\prime} a$ & $n a b ' a i$ & 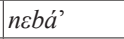 & $n a b a$ & $n a b a$ & $n a b a^{\prime}$ & $n a a b ' a$ \\
\hline $93 \mathrm{~b}$. & & & & & $n a^{\prime} a$ & & & \\
\hline $93 \mathrm{c}$. & & & & & & sara & & \\
\hline 93d. & & & & & & & & hami \\
\hline 94. water & yam & yam & yam & yam & yam & yum & yam & yam \\
\hline 95. we & hanán/-ín & henen & hinin & hanćn & hanin & hini & hinin & hinin \\
\hline 96. what & $n a \bar{n}(a) ?$ & $n \bar{a} ?$ & naanaati & nan & $n \bar{a}$ & naa & naan & naan \\
\hline 97a. white & éra / éla & cra / cla & eerabi & eeráb & $\bar{e} r a b / \bar{e} l a b$ & é:rāb/é:lāb & eeraab/t & $\begin{array}{l}\text { eera / } \\
\text { eela } \mathrm{dim} .\end{array}$ \\
\hline 97b. & & & & & 'adaro/-l- ${ }^{+}$ & adaro,-lo & & \\
\hline 98. who & $a w ?$ & $\begin{array}{l}a w ? \\
\text { acc. } \bar{a} b \\
\text { gen. } \bar{a} y\end{array}$ & $a a b i$ & $a a b$ & $\begin{array}{l}a u \\
a y \\
\end{array}$ & $\begin{array}{l}a \bar{u} \\
\bar{a} b u \text {, } \\
\bar{a} i \text { whose }\end{array}$ & $\begin{array}{l}a a w \\
a a b \\
a a y i\end{array}$ & $a w$ \\
\hline 99a. woman & takát & takat & takat & $t \varepsilon k \varepsilon \varepsilon t$ & takat & takat & takatt & takat \\
\hline $99 \mathrm{~b}$. & & & & & pl. $m a$ & & & pl. $m^{\prime} a$ \\
\hline $99 \mathrm{c}$. & & & & & & & & $\begin{array}{l}\text { (kwaat female } \\
\text { cf. } k w a \text { sister) }\end{array}$ \\
\hline 100a. yellow & & & & & $\begin{array}{l}(\text { dērūb/-l- } \\
\text { brown) }\end{array}$ & & & - \\
\hline $100 \mathrm{~b}$. & $\underline{a ́ s f a r}$ & & & & & asfar & & \\
\hline $100 \mathrm{c}$. & & ádarsótai & & & & & & \\
\hline 100d. & & clay & & & & & & \\
\hline $100 \mathrm{e}$. & & & kwirkumi & & & kurkum & & \\
\hline
\end{tabular}

Appendix 2. Old and fragmentary wordlists

\begin{tabular}{|c|c|c|c|c|c|c|c|c|}
\hline & Seetzen & $\begin{array}{c}\text { Salt } \\
{ }^{\mathrm{K}} \text { Krockow }\end{array}$ & $\begin{array}{l}\text { Burckhart } \\
\text { LLinant }\end{array}$ & $\begin{array}{l}\text { Kremer } \\
\text { LuLucas }\end{array}$ & Thompson & $\begin{array}{l}\text { Watson } \\
{ }^{\mathrm{H}} \text { Huber }\end{array}$ & Munzinger & Almkvist \\
\hline 1a. all & & ${ }^{\mathrm{K}} \operatorname{cass}[\mathrm{o}]$ & & kassó & & & $\operatorname{kess}[a]$ & $\operatorname{kar}(i)_{S}$ \\
\hline 2a. ashes & netásch & & & & & & net hash & nètháás \\
\hline $2 \mathrm{ac}$. & & & & néed-dôl & & & & \\
\hline 3a. bark & & & & & & & te'edf & ádif \\
\hline $3 \mathrm{~b}$ & & & & & & & o'demo & \\
\hline 4a. belly & [o]phéh & & ofy & & oofé & & $o^{\prime} f i$ & $f i, f e$ \\
\hline
\end{tabular}




\begin{tabular}{|c|c|c|c|c|c|c|c|c|}
\hline & Seetzen & $\begin{array}{c}\text { Salt } \\
{ }^{\mathrm{K}} \text { Krockow }\end{array}$ & $\begin{array}{c}\text { Burckhart } \\
\text { LLinant }\end{array}$ & $\begin{array}{l}\text { Kremer } \\
\text { LuLucas }\end{array}$ & Thompson & $\begin{array}{l}\text { Watson } \\
{ }^{\mathrm{H}} \text { Huber }\end{array}$ & Munzinger & Almkvist \\
\hline $4 \mathrm{~b}$. & & $\begin{array}{l}\text { o calaho } \\
{ }^{\mathrm{K}}[\text { eh] } \text { kalawah }\end{array}$ & & & & & & kálawa \\
\hline 5a. big & wuinnu[u] & win & & uenn [u] & & & wuun & win \\
\hline 6a. bird & [o]klá & kelai & kilay & ókla & oklaï & & to'kelei & $k(e) l^{\prime} a$ \\
\hline 7a. bite & phinnik- & & & & & & éfnek & fénik \\
\hline 8a. black & haddál & $\begin{array}{l}\text { ([o]hadal } \\
\text { brun) }\end{array}$ & $\begin{array}{l}\text { haddal } \\
\mathrm{L}_{\text {wohaddul }}\end{array}$ & haddal & & & haTel & hádal \\
\hline $8 \mathrm{~d} .=35$ & & & $\mathrm{~L}_{\text {sotago }}$ & & & & & \\
\hline 9. blood & [o]óih & & [o]boy & & & & o'boi & $b \overline{o y}$ \\
\hline \multirow[t]{2}{*}{ 10. bone } & [t]mitât & & [ti]mita & & pl. te-mitât & & to 'mitát [t] & 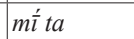 \\
\hline & & & & & $\operatorname{miak}^{u} a$ & & & \\
\hline 11b. breast & onúk & & & & & & o'nug & $n \bar{u} g$ \\
\hline $11 \mathrm{c} .=40$ & [ê]gná & & & & & & & \\
\hline $11 \mathrm{~d}$ & & & & $a d t a b a$ & o-dăbá & & & \\
\hline $11 \mathrm{~g}$ & & & & & & & te'gibab & \\
\hline 12a. burn & & & & & & & elú & $l \bar{u}, l u w$ \\
\hline 13a. claw & [e]néf & & & & & & o'naff & naf \\
\hline 14a. cloud & & & & & & & o'lesso & \\
\hline $14 \mathrm{~b}$ & & & & & & & & áfrat \\
\hline $14 \mathrm{c}$. & & & & & & & & $\check{s} a y / \check{s} w a$ \\
\hline $14 \mathrm{i}$. & saháb & & & & & & & \\
\hline 15a. cold & & & $\begin{array}{l}\text { ([tu]la } \\
\text { warm!) }\end{array}$ & & & & la & lé'a \\
\hline $15 \mathrm{c}$ & & mocquara & & & $m a k^{u} i r$ & & & \\
\hline 16a. come & & & & & & & & $\bar{l}$ \\
\hline $16 \mathrm{~b}$. & & & & & & máa & & má'a! \\
\hline 17a. die & & & & & & toyét & -yayá- & $y a(i)$ \\
\hline 18a. dog & oyás & woyas & oyas & $\begin{array}{l}\text { óyas } \\
\text { Lu wayas }\end{array}$ & & oyás & o'yas & $y \bar{a} s$ \\
\hline 19a. drink & guanéh 1sg. & & goa & gua & & & gúye & $g^{w} a$ \\
\hline 20a. dry & & & & & & & $e \check{s} a$ & \\
\hline $20 \mathrm{~b}$ & & & & & & & & bélama \\
\hline 21. ear & [w]ongwil & ongueloh & [to]ngy & oónquil & & wankuil & o'anguil & 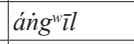 \\
\hline 22a. earth & & tobút & & & & & $\begin{array}{l}\text { to'but, pl. } \\
\text { te'bura }\end{array}$ & $b \bar{u} r$ \\
\hline $22 \mathrm{c} . ?$ & & & & totayáh & & & & \\
\hline 23a. eat & támanéh & & $\operatorname{tam}[a]$ & & & tama & damya & tam \\
\hline 24. egg & [o]keh & & & & & & $k u h i(b)$ & $k u ́ h i / k^{w} a ́ h i$ \\
\hline 25a. eye & egoát & & & & & & o'guedj & \\
\hline $25 \mathrm{~b}$. & & telele & tilyly & teléle & & telil & & líli \\
\hline 26a. fat n. & tószmûm & & & & & & semúm & \\
\hline $26 \mathrm{~b}$. & & & & & & & & $l a^{\prime}$ \\
\hline $26 \mathrm{~d}$ & & & Lto omfou & & & & & \\
\hline 27. feather & & & & & & & anbor & $\begin{array}{l}\text { (ánbūr } \\
\text { wing) }\end{array}$ \\
\hline 28. fire & {$[t \hat{\jmath}] n-i h$} & $\begin{array}{l}\text { to ne } \\
\mathrm{\kappa} \text { dohnè }\end{array}$ & {$[$ to]ney[t] } & tona & & twané & to'ne & $n a$ \\
\hline \begin{tabular}{|l|} 
26a. fish \\
\end{tabular} & & & & & & & & $\underline{h \bar{u} t}$ \\
\hline $29 \mathrm{~b}$. & uâscho & wa assu & & & & & & \\
\hline 30a. fly v. & & & & & & & ferya & fir \\
\hline
\end{tabular}




\begin{tabular}{|c|c|c|c|c|c|c|c|c|}
\hline & Seetzen & $\begin{array}{c}\text { Salt } \\
{ }^{\mathrm{K}} \text { Krockow }\end{array}$ & $\begin{array}{c}\text { Burckhart } \\
\text { LLinant }\end{array}$ & $\begin{array}{l}\text { Kremer } \\
{ }^{\text {LuLucas }}\end{array}$ & Thompson & $\begin{array}{l}\text { Watson } \\
{ }^{\mathrm{H}} \text { Huber }\end{array}$ & Munzinger & Almkvist \\
\hline $30 \mathrm{~b}$. & & & & & & & & $b \bar{i} r$ \\
\hline 31a. foot & & & & & & & te’sókena & \\
\hline $31 \mathrm{~b}$. & reggét & rugădok & ragad & ragad & & reked & & ragád \\
\hline $31 \mathrm{~d}$. & & & ${ }^{\mathrm{L}} \mathrm{O}$ andarthe & & & & & \\
\hline $31 \mathrm{c}$. & $\begin{array}{l}\text { ([te]démbe } \\
\text { sole) }\end{array}$ & & & tadembé & & & $\begin{array}{l}\left(e^{\prime} \text { dembi }\right. \\
\text { calf })\end{array}$ & $\begin{array}{l}\text { (dámba } \\
\text { sole })\end{array}$ \\
\hline $31 \mathrm{e}$. & & & & & $o-s ̌ a w a$ & & & \\
\hline 32. full & $a t t a ̂ b[t o]$ & & & $a t a b[t]$ & & & & átāb \\
\hline 33a. give & hitôkeneh & & & & & hiá & & $h i \& a u$ \\
\hline 34b. good & & & & & & & shebób & šebób \\
\hline 34b. good & $\begin{array}{l}\text { (dái[bo] } \\
\text { gesund) }\end{array}$ & $\begin{array}{l}a \text { daiuwa } \\
{ }^{\mathrm{K}} \text { daib }\end{array}$ & & & & $\begin{array}{l}\text { dayé, } \\
\text { dayíb }\end{array}$ & $d a i$ & $d \bar{a} y$ \\
\hline 35. green & & $\begin{array}{l}\text { (sotago } \\
\text { black) }\end{array}$ & $\begin{array}{l}\text { ('osotăy } \\
\text { yellow) }\end{array}$ & ssóto & & & & sốtay \\
\hline 36a. hair & [ta]hamú & $\begin{array}{l}\text { tamo } \\
\mathrm{K}[t] \text { ammeh }\end{array}$ & $\begin{array}{l}\text { ([e]hamo } \\
\text { wool) }\end{array}$ & [te]háma & & tahmé & te'hamo & hámu \\
\hline 37e. hand & tedembetôn & & & & $\begin{array}{l}\text { (te-dumbê } \\
\text { palm of } \\
\text { hand) }\end{array}$ & & & \\
\hline $37 \mathrm{a}$. & & & oya & 'uaya & & ayeb & o'eye & ay \\
\hline 38a. head & ógürmá & $\begin{array}{l}\text { iggremă } \\
\left({ }^{\mathrm{K}} \text { girmah }\right. \\
\text { front })\end{array}$ & & gurma & & akermá & o'agurma & gúrma \\
\hline 39. hear & & & $\begin{array}{l}\text { [te]msyra } \\
\mathrm{L} \text { emsiwoh }\end{array}$ & & & & omásu & $m \dot{a} s s u$ \\
\hline 40a. heart & ginna- & ${ }^{\mathrm{K}}[$ eh]gnah & & & & & o'gena & gína \\
\hline 41a. horn & {$[o] d a$} & & & & & & & \\
\hline 42. I & & & & ane & & $a n e b$ & ane & áne \\
\hline 43. kill & addirro & $\operatorname{dir}(-n a)$ & dera & & & & $\bar{e} d e r$ & der, dir \\
\hline 44a. knee & & Koh gummba & & & & & o'gumba & \\
\hline $44 \mathrm{~b}$. & [e]géndef & & & & & & & gúnduf \\
\hline 45. know & & Kektenah & & & & & \begin{tabular}{|l} 
ékdēn \\
kenan $\mathrm{n}$.
\end{tabular} & kan \\
\hline 46a. leaf & tolât & & & & & & to'rat & \\
\hline \multicolumn{9}{|l|}{ 47. lie } \\
\hline 48a. liver & tôszéh & & & & tó-si & & to $s \bar{e}$ & sa \\
\hline 49a. long & $\begin{array}{l}\text { (gummet[to] } \\
\text { tief) }\end{array}$ & & & & & & gemed & gúmad \\
\hline 50a. louse & & & & & & & to'se & \\
\hline $50 \mathrm{~b}$. & totât & & & & & & to'tat & $t a \bar{t}$ \\
\hline 51a. man & oták & $\begin{array}{l}\text { gal-tuk } \\
\mathrm{K}[\mathrm{o} \text { ]teek }\end{array}$ & [o]tak & & & téki & o'tekk & tak \\
\hline 52. many & guda[bo] & $\begin{array}{l}\text { guddowa } \\
{ }^{\mathrm{K}} \text { guddah }\end{array}$ & & & & & guda $[b]$ & $g \stackrel{\bar{u}}{d} d a$ \\
\hline 53a. meat & tôschá & K[doh]sharr & [to]sha & toscha & & & to'sha & $\check{s} a$ \\
\hline 54a. moon & totríg & & & eterri $(g)$ & & totrek & o 'édrik & térig \\
\hline $54 \mathrm{~b}$ & & te dai & ondjim & & & & & \\
\hline $\begin{array}{l}55 a . \\
\text { mountain }\end{array}$ & {$[o] r b a$} & $\begin{array}{l}\text { orba } \\
\text { Kórba }\end{array}$ & [o]rbay & órba & & & o'orba & réba \\
\hline 56. mouth & Lo]yéf & & [o]yaf & oyeff & & oyéf & o'yeff & yef, yaf \\
\hline 57. name & & & & & & & o'sem & $\operatorname{sim}$ \\
\hline
\end{tabular}




\begin{tabular}{|c|c|c|c|c|c|c|c|c|}
\hline & Seetzen & $\begin{array}{c}\text { Salt } \\
{ }^{\mathrm{K}} \text { Krockow }\end{array}$ & $\begin{array}{c}\text { Burckhart } \\
\text { LLinant }\end{array}$ & $\begin{array}{l}\text { Kremer } \\
\text { LuLucas }\end{array}$ & Thompson & $\begin{array}{l}\text { Watson } \\
{ }^{\mathrm{H}} \text { Huber }\end{array}$ & Munzinger & Almkvist \\
\hline 58a. neck & & & & & & & tále & ále \\
\hline $58 \mathrm{~b}$. & [em]magéh & & & & & & & máge \\
\hline $58 \mathrm{c}$. & & & ${ }^{\mathrm{L}}$ to $m \hat{o} c$ & {$[t o] m o(k)$} & & & & $m \bar{k} k$ \\
\hline 59. new & geyi[bo] & & & & & & $g i$ & $g \frac{a}{a} i$ \\
\hline 60a. night & [wu]hauâd & ouad & & & & & o'hauad & hawàd \\
\hline 61. nose & [o]gnûf & ognuf & [to]genouf & [o]gnuff & & kenuf & & genúff \\
\hline 62b. not & & & & & & & $\begin{array}{l}\text { kike } \\
\text { es ist nicht }\end{array}$ & \\
\hline $62 \mathrm{c}$. & & & ${ }^{\mathrm{L}}$ lano & & & & & (lau nein) \\
\hline 63. one & $n k a ̂ l$ & en-gat & engaro & $n g a(t)$ & & kal & engar/l,-t & $\begin{array}{l}\text { (en)gāl m. } \\
\text { (en)gāt f. }\end{array}$ \\
\hline 64a. person & & & & & & & & ádame \\
\hline $64 \mathrm{c}$ & odéiy & & & & & & & \\
\hline 65a. rain $\mathrm{n}$. & [ô]bre & o berrah & $\overline{o b r a}$ & óbra & & & o'berē & bire \\
\hline 66. red & addaro & wo druf & adaro [b] & & & & aderō & ádarō \\
\hline 67a. road & déreb & & & & & & & déreb \\
\hline $67 \mathrm{c}$ & & & ${ }^{\mathrm{L}}$ osala & & & & & \\
\hline $67 \mathrm{e}$. & & & & & & pl. ${ }^{\mathrm{H}} d \bar{o} i a ̆$ & & \\
\hline 68a. root & & & & & & & gedem & \\
\hline 69a. round & & & & & & & debala & debálu \\
\hline $69 \mathrm{~b}$ & kwállalábo & & Lqualal[ho] & & & & & $k^{w}$ alál \\
\hline 70a. sand & & & & & & & & dába \\
\hline 71a. say & & & & & & & $d i$ & $d i$ \\
\hline $71 \mathrm{~b}$. & & & & & & & soiya & $s \bar{o}$ \\
\hline 72a. see & & & & & & & rehya & rēh \\
\hline $72 b$ & & & $\begin{array}{l}\text { shebabo } \\
{ }^{\mathrm{L}} \text { chebbat }\end{array}$ & & & & eshib & šébib \\
\hline 73a. seed & tarteh & & & & & & & \\
\hline 74a. sit & & & $s a[a ́]$ & & & $s a ́$ & esá & $s a^{\prime}$ \\
\hline 75a. skin & $\begin{array}{l}\text { [wu]adéh } \\
\text { [t]ade[to] }\end{array}$ & ${ }^{\mathrm{K}}$ worhade & $\begin{array}{l}\left({ }^{\mathrm{L}} \text { to hadah }\right. \\
\text { body) }\end{array}$ & & & & o'ade & áde \\
\hline $75 \mathrm{~b}$ & & & $\begin{array}{l}\text { osar } \\
{ }^{\mathrm{L}} \text { o serre }\end{array}$ & & & & & sar \\
\hline 76a. sleep & dauádenéh & & & ana duane & & & duya & $d \bar{u}$ \\
\hline 77a. small & & & & & & & & des \\
\hline $77 \mathrm{~b}$ & dábaló[bu] & $\begin{array}{l}\text { dubalowa } \\
\text { dabàloh }\end{array}$ & & tabalo[b] & & & & dábal/r/no \\
\hline $\begin{array}{l}\text { 78a. smoke } \\
\text { n. }\end{array}$ & [wu]êga & & & & $o-d \hat{e}$ & & o'ège & éga \\
\hline 79. stand & & & & & & kéda & enget & (é) $\dot{n g a d}$ \\
\hline 80. star & iéheiyúk & haiek & & hayúk & & whayok & o'heyok & hayük \\
\hline 81. stone & [wu]auiy & $\begin{array}{l}\text { owee } \\
\mathrm{K}_{\text {}} \text { wauèh }\end{array}$ & awey & & & & o'auē & áwe \\
\hline 82. sun & toin & to een & toyn & toi & tô-i, to-yin & toïn & to'êin & ìn / yin \\
\hline 83a. swim & & & & & & & beddefya & \\
\hline $83 \mathrm{~b}$. & & & & & & & & $\underline{\bar{u} m}$ \\
\hline $83 \mathrm{c}$. & & & & & nidăba & & & \\
\hline 84a. tail & & & & & & & enníwa & níwa \\
\hline $84 b$ & [wu]haggeh & & & & & & & \\
\hline 85. that & & & & & & & & $b \bar{e}-n /-t$ \\
\hline
\end{tabular}




\begin{tabular}{|c|c|c|c|c|c|c|c|c|}
\hline & Seetzen & $\begin{array}{c}\text { Salt } \\
{ }^{\mathrm{K}} \text { Krockow }\end{array}$ & $\begin{array}{c}\text { Burckhart } \\
\text { LLinant }\end{array}$ & $\begin{array}{l}\text { Kremer } \\
\text { LuLucas }\end{array}$ & Thompson & $\begin{array}{l}\text { Watson } \\
{ }^{\mathrm{H}} \text { Huber }\end{array}$ & Munzinger & Almkvist \\
\hline $\begin{array}{l}\text { 86. this sg. } \\
\text { m. : f. pl. }\end{array}$ & & & & & & & & $\begin{array}{l}\bar{u} n: t \bar{u} n \\
\bar{a} n: t \bar{a} n\end{array}$ \\
\hline 87. thou & & & & & & berok & & $\begin{array}{l}\text { barük } \mathrm{m} . \\
\text { bātúuk } \mathrm{f} .\end{array}$ \\
\hline 88. tongue & [e]midap & medăbo & ${ }^{\mathrm{L}} O$ midab & mída & & imidab & o'miḍala[b] & mida \\
\hline 89a. tooth & tékoréh pl. & K[deh] kurre & ${ }^{\mathrm{L}}$ to courah & togura & to-kurrê & & $\begin{array}{l}\text { to'kole, } \\
\text { pl. te kore }\end{array}$ & kóra \\
\hline $89 \mathrm{~b}$. & & & & & & ekosé & & \\
\hline 90a. tree & uhinde & & hindy & ohindi & & & o'hindi & hinde \\
\hline $90 \mathrm{~b}$. & & ${ }^{\mathrm{K}}$ gahl & & & & & & \\
\hline 91. two & malloba & máloob & molobo & maló & & maluba & meló & malób \\
\hline 92a. walk & & & & & & & béya & bay \\
\hline $92 \mathrm{~b}$. & & & & & giga (men) & gige & gígya & $g \bar{l} g$ \\
\hline 92c. & & & saka & sakká & saka (dogs) & saká & sakya & sak \\
\hline 93a. warm & nebba & & Lnabah[o] & & & & neba & néba' \\
\hline $93 \mathrm{c}$ & & houeet & & & & & & \\
\hline 94. water & êyem & o yum & ayam & $\begin{array}{l}\text { éyam } \\
\text { Luaeer! }\end{array}$ & & yém & o'yem & $y \overline{o m}$ \\
\hline 95. we & & & & & & henena & & hénen \\
\hline 96. what & & & ${ }^{\mathrm{L}}$ nan- & & & nanaté & & $n \bar{a} n(a)$ \\
\hline 97a. white & $\begin{array}{l}\text { (erab }[o] \\
\text { licht })\end{array}$ & & & & & & era & érā \\
\hline $97 \mathrm{c}$. & & & wodana & & & & & \\
\hline 98. who & & & & & & $a^{\prime} a b$ & & $a u$ \\
\hline $\begin{array}{l}\text { 99a. } \\
\text { woman }\end{array}$ & $\begin{array}{l}\text { (tetakkát } \\
\text { Weib) }\end{array}$ & $\begin{array}{l}\text { tukut } \\
{ }^{\mathrm{K}} \text { takat }\end{array}$ & [ta]taket & & & tekét & te'tekék & tákat \\
\hline $99 \mathrm{~b}$ & & & & & & & te'ma & pl. $m a$ \\
\hline 100b. yellow & & & & & & & & ásfar \\
\hline $100 f$. & & & & teta $(b b a)$ & & & & \\
\hline $100 \mathrm{~g} .=35$ & & & ${ }^{\mathrm{L}}$ osotăy & & & & & \\
\hline
\end{tabular}

Note: The underlined words are loans. The round brackets indicate the etymologically related words, but with shifted semantics. Such parallels were not included in calculation.

\section{CONCLUSION}

In the present study the core lexicon of Beja (Bedawie) is collected from both (almost) complete or modern and from fragmentary or outdated records. From them ten richest sources served as a base of the lexicostatistic comparison with a glottochronological interpretation. The result, i.e. the beginning of disintegration dated to the early 9 th century (Tree Diagram 1), is fully comparable with the time depth of the Oromo dialect continuum (Tree Diagram 2). On the other hand, it is apparent that the correlation between almost complete lexicons with synonyms is higher in comparison with the wordlists (Bender, Thelwall) or incomplete lexicons (Munzinger, partially Almkvist, Starkey), where the results are a little distorted. It is also necessary to take into account that some authors included in their lexicons the data of some of their predecessors (Almkvist, Reinisch, Wedekind), not always with indication. So, if two most incomplete or deviant sources, namely Munzinger 
and Bender, are excluded, the common share for the remaining idioms is c. $95 \%$ or higher. It implies the beginning of their disintegration around AD 1200. This younger result better agrees with at least partial intelligibility between the tribal dialects of Beja.

\section{ACKNOWLEDGMENT}

First of all, I would like to express my gratitude to Martine Vanhove, who kindly mediated her unpublished lexical data for this study and corrected my English, and to Andrzej Zaborski, who first drew my attention to Beja. Thanks to Bernd Heine and Heinz-Jürgen Sasse I had a unique chance to collect the data for the Etymological Dictionary of Beja in Cologne in 1993-94 and 1998. The recalibrated glottochronology used here was developed by Sergei Starostin, who was very interested in research of Beja. The final version of this contribution originated thanks to the grant of the The Czech Science Foundation (GAČR), P406/12/0655.

\section{REFERENCES}

Almkvist H. 1881-1885. Die Bischari-Sprache (Tu Bedawie) in Nordost-Afrika I-II. Uppsala-Berlin.

Bender M. Lionel. 1971. "The Languages of Ethiopia. A New Lexicostatistic Classification and Some Problems of Diffusion." Anthropological Linguistics 13(5), 165-288.

Bender M. Lionel. 1976. The Non-Semitic Languages of Ethiopia. East Lancing: Michigan State University.

BlAžEK Václav. 2010. "Glottochronological Classification of Oromo Dialects." Lingua Posnaniensis 52(2), $27-42$.

BurCKHARDT J.L. 1819. Travels in Nubia. London: Murray.

Heuglin M.T. von 1858. Reise in Nordost Afrika und längs des Rothen Meeres. Gotha.

Hudson Richard A. 1964. A Grammatical Study of Beja. London: PhD. Diss. of the School of Oriental and African Studies.

Hudson Richard A. 1976. "Beja." In: Bender 1976: 97-132.

Hudson Richard A. 1996. A Dictionary of Beja. Cambridge: Ms. prepared by Roger Blench.

Kremer A. von. 1863. Aegypten: Forschungen über Land und Volk während eines zehnjährigen Aufenthalt. I. Leipzig: Brockhaus.

Krockow C. von. 1867. Reisen und Jagden in Nordost-Afrika. II. Berlin.

LinANT De Bellefonds Louis M.A. 1868. L'Etbaye, pays habité par les Arabes Bicharieh. Géographie, ethnologie, mines d'or. Paris: Bertrand.

LuCAS L. 1876. "On Natives of Suakin and Bishareen Vocabulary." The Journal of the Anthropological Institute of Great Britain and Ireland 6(II), 191.

MeINHOF Carl. 1919. "Sprachstudien im egyptischen Sudan. D. Eine Hamitensprache: 44. Bedauye." Zeitschrift für Kolonialsprachen 9, 246-255.

Morin Didier. 1995. "Des paroles douces comme la soie". Introduction aux contes dans l'aire couchitique (bedja, afar, saho, somali). Paris: Peeters.

MunZINGer Johann A.W. 1864. Ostafrikanische Studien. Schaffhausen.

ReINISCH Leo. 1893-1894. “Die Bed̦auye-Sprache in Nordost-Afrika, II. and III.” Sitzungsberichte der Akademie der Wissenschaften, Philol.-hist. Classe 128(VII), 130(VII).

REINISCH Leo. 1895. Wörterbuch der Bedauye-Sprache. Wien: Hölder.

Renfrew C., McMahon A., Trask L. 2000. Time Depth in Historical Linguistics. Vol. 1. Cambridge: McDonald Institute for Archaeological Research, 223-265.

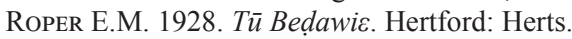

SALt Henry 1816. Voyage en Abyssinie. Paris: Imprimerie de Demonville.

SCHULER E. von. 1989. XXIII. Deutscher Orientalistentag (Würzburg 1985). Stuttgart: Steiner.

SeEtZen U.J. apud Vater J.S. 1816. Proben Deutscher Volks-Mundartan. Dr. Seetzen's linguistischer Nachlass und andere Sprachforschungen und Sammlungen, besonders über Ostudien, Herausgegeben. Leipzig: Gerhard Fleischer.

SHevoroshrin Vitaly, Sidwell Paul. 1999/2000. Historical Linguistics \& Lexicostatistics. Melbourne: Association for the History of Language, Science \& History of Languages 3, 3-50.

Starkey Janet. n.d. Amarar Lexicon. University of Durham: Ms. 
Starostin Sergej. 1999/2000. "Comparative-historical Linguistics and Lexicostatistics." In: ShevoroshKIN \& Sidwell 1999/2000: 3-50; also in: ReNFrew et al. 2000: 223-265.

Thelwall Robin E.W. 1970. Linguistic Investigations in the Sudan: Beja Wordlist. Khartoum: Ms.

Thompson C. 1910. "Some Hadendoa Words hitherto unpublished." Man 95, 164-167, 99-100, 180-182.

Vanhove Martine. 2006. "The Beja Language Today in Sudan: The State of the Art in Linguistics." In: Proceedings of the 7th International Sudan Studies Conference, April 2006, Bergen, Norway. Bergen: CD Rom (University of Bergen), http://halshs.archives-ouvertes.fr/docs/00/06/52/11/PDF/Beja_State_of_the_Art_ Bergen.pdf.

VANHOVE Martine. 2008. Field records from Sinkat (Sudan). Unpublished lexical data kindly offered to the author.

Watson C.M. 1888. Languages Spoken at Suakin: Arabic, Hadendoa, Beni-Amer. London: Society for Promoting Christian Knowledge.

WedEKInd Klaus \& Charlotte, Musa Abuzeinab. 2007. A Learner's Grammar of Beja (East Sudan). Grammar, Texts and Vocabulary (Beja-English and English-Beja). Köln: Köppe.

ZABORSKI Andrzej. 1989. "Der Wortschatz der Bedscha-Sprache - eine vergleichende Analyse." In: SchUlER 1989: 573-591. 